\title{
Suppression of Evil promotes the osteogenic differentiation and inhibits the adipogenic differentiation of bone marrow-derived mesenchymal stem cells in vitro
}

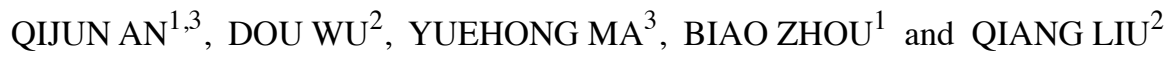 \\ ${ }^{1}$ Shanxi Medical University, Taiyuan, Shanxi 030001; ${ }^{2}$ Department of Orthopaedics, \\ Shanxi Dayi Hospital, Shanxi Academy of Medical Sciences, Taiyuan, Shanxi 030032; \\ ${ }^{3}$ Shanxi Provincial People's Hospital, Taiyuan, Shanxi 030012, P.R. China
}

Received January 1, 2015; Accepted October 14, 2015

DOI: $10.3892 / \mathrm{ijmm} .2015 .2385$

\begin{abstract}
Osteoporosis (OP) is considered a complex disease with a strong genetic impact, mainly affecting post-menopausal women and is also a common cause of fracture. Elucidating the molecular mechanisms that regulate the osteogenic differentiation of bone marrow-derived mesenchymal stem cells (BMSCs) is crucial to developing treatment strategies to combat OP. In the present study, we found that ectopic viral integration site-1 (Evil) was highly expressed during the process of adipogenesis of rat BMSCs. Notably, Evil levels markedly increased on day 3 of adipogenic differentiation following the addition of adipogenic induction supplements. In addition, we interfered with the expression of the Evil gene in the adipogenesis of BMSCs by supplementing adenoviral plasmids and measured the expression levels of bone sialoprotein (BSP), osteocalcin ( $\mathrm{OCN})$, osteopontin (OPN), peroxisome proliferator-activated receptor $\gamma 2$ (PPAR $\gamma 2$ ) and lipoprotein lipase (LPL) by RT-qPCR and western blot analysis. The mRNA and protein levels of osteogenic and adipogenic markers in the BMSCs were up- and downregulated, respectively following the silencing of siEvil. Our experimental results substantiate that the suppression of Evil in BMSCs by RNA interference inhibits adipogenic differentiation, while it promotes osteogenic differentiation. The results from our study demonstrated that the Evil gene may be targeted as a therapeutic strategy for promoting bone formation.
\end{abstract}

Correspondence to: Professor Qiang Liu, Department of Orthopaedics, Shanxi Dayi Hospital, Shanxi Academy of Medical Sciences, 99 Longcheng Da Jie, Taiyuan, Shanxi 030032, P.R. China E-mail: qiangliu5719@163.com

Abbreviations: BMSCs, bone marrow-derived mesenchymal stem cells; Evi1, ectopic viral integration site-1; BSP, bone sialoprotein; OPN, osteopontin; OCN,osteocalcin;PPAR $\gamma$,peroxisomeproliferatoractivated receptor $\gamma$; LPL, lipoprotein lipase

Key words: Evi1, bone marrow-derived mesenchymal stem cells, osteoporosis, adipogenic differentiation, RNA interference

\section{Introduction}

Osteoporosis (OP), a major health concern of post-menopausal women and the elderly, is a common skeletal disorder characterized by low bone mass and microarchitectural deterioration of bone tissue with increased susceptibility to fracture $(1,2)$. Advanced age, gender and immobilization are major risk factors for developing OP besides a series of other contributors, such as diminished gender steroid production in elderly individuals and following menopause $(3,4)$.

Emerging evidence has indicated that $\mathrm{OP}$ is associated with stem cell defects $(5,6)$, including osteoblast progenitors [mesenchymal stem cells (MSCs)] residing in the bone marrow (BM) (7). Bone marrow-derived mesenchymal stem cells (BMSCs), a class of multipotent and self-renewing cells that give rise to differentiated progeny when implanted into appropriate tissues, have emerged as a particularly appealing option over the last decade. Pittenger et al found that BMSCs, the precursor cells of adipocytes and osteoblasts (8), play an important role in bone physiology and partly participate in the pathophysiology of OP. Furthermore, as previoulsy demonstrated the bone mass and strength of osteoporotic rats and mice can be restored through direct BMSC injection by local or systemic transplantation (9-11).

BMSCs are more sensitive and more effective towards osteogenic differentiation than adipose tissue-derived stem cells (ADSCs) (12), another emerging type of stem cells for treating OP, which is relatively abundant and can be easily harvested through simple methods, such as lipoaspiration or surgical resection (13).

A previous study demonstrated that the osteogenic differentiation ability of BMSCs is lower in post-menopausal women suffering from OP compared to pre-menopausal women (14). Furthermore, in another previous study, an augmented volume of adipose tissue was found in the BM of post-menopausal women, indicating that the ability of BMSCs to differentiate into adipocytes was enhanced (7). Theoretically, there is an inverse association between osteogenic and adipogenic lineage commitment and differentiation; that is to say, differentiation towards an adipocytic phenotype occurs at the cost of an osteoblast phenotype. 
In the present study, we examined the initiation and adipogenic differentiation of cultured BMSCs at the cellular level. We hypothesized that ectopic viral integration site-1 (Evi1), a key regulator in the process of the adipogenic differentiation of 3T3-L1 preadipocytes (15), would lead to the adipogenic differentiation of BMSCs in vitro. To the best of our knowledge, the expression of Evil in BMSCs has not been reported to date. We hypothesized that the suppression of Evil by RNA interference (RNAi) would mediate the expression of specific osteogenic and adipogenic genes and become a drug target for the treatment of OP.

\section{Materials and methods}

Isolation of BMSCs and cell culture. BMSCs were isolated and cultured in vitro and passaged using a cell adherent culture method from the BM of Sprague-Dawley (SD) rats, as previously described (16). Briefly, rat BM was obtained according to the research guidelines and under the approval of Shanxi Medical University (Shanxi, China). Healthy specific pathogen-free (SPF) grade 4-week-old SD rats (weighing $100-120 \mathrm{~g}$ ) were sacrificed by an intraperitoneal injection of pentobarbital sodium. The bilateral femurs and tibias were harvested under aseptic conditions and all soft tissues were removed. Metaphyses from both ends were resected and BM cells were collected by flushing the BM cavity in a sterile petri dish with Dulbecco's modified Eagle's medium (DMEM; Gibco, Shanghai, China) containing $10 \%$ fetal bovine serum (FBS; Gibco, Carlsbad, CA, USA) and antibiotics (100 U/ml penicillin and $100 \mu \mathrm{g} / \mathrm{ml}$ streptomycin). Cells isolated from each rat were inoculated in two $25 \mathrm{~cm}^{2}$ flasks. The medium was first changed $24 \mathrm{~h}$ after inoculation to remove the unattached cells, and then twice each week until confluence was achieved. The BMSCs were cultured at $37^{\circ} \mathrm{C}$ in a $5 \% \mathrm{CO}_{2}$-supplemented incubator and were capable of differentiating into adipogenic and osteogenic cells under specific induction, as previously described (17).

The differentiation of cultured BMSCs was monitored with Oil Red O staining (on day 16) for terminal adipocyte differentiation and with alizarin red staining (on day 16) for terminal osteoblast differentiation.

Cells from the 3rd passage were used for the experiments. BMSC adipogenesis was induced at confluence with the use of an induction medium of 10\% FBS in DMEM supplemented with penicillin/streptomycin (P/S), $200 \mu \mathrm{M}$ indomethacin, $1 \mu \mathrm{M}$ dexamethasone, $0.5 \mathrm{mM}$ isobutylmethylxanthine and $0.5 \mu \mathrm{g} / \mathrm{ml}$ insulin (all from Sigma, St. Louis, MO, USA) for 21 days, and the medium was changed twice each week. RNA and protein were isolated within the first 10 days. Transient transfections were performed with the use of adenovirus.

Vector construction and transfection of the cells. Short hairpin RNA (shRNA) encoding DNA sequences were synthesized by Invitrogen Life Technologies (Carlsbad, CA, USA) and constructed into adenoviral plasmids, and adenoviruses were prepared according to previously described procedures (18). The sequence of shRNA against luciferase was 5'-CTTACG CTGAGTACTTCGA-3'; shRNA against Evi1 was (shEvi1-1) 5'-GGAAGCAACATGGAAACAA-3' and (shEvi1-2) 5'-GCA GTGAGGTCTGCCATAA-3'. We created three adenoviral vectors, termed Ad-shEvi1-1 (KD1), Ad-shEvil-2 (KD2) and AD-shSc, respectively. KD1 and KD2 encoding Evil shRNA were used in the experimental groups. AD-shScr expressing scrambled shRNA was used in the mock group and the control group was untransfected cells.

The cells were inoculated into $60-\mathrm{mm}$ dishes in $10 \%$ FBS-DMEM and cultured until nearly $90 \%$ confluence and were then incubated in complete serum-free medium for $12 \mathrm{~h}$. Subsequently, the cells were treated with shEvil or shScr (scrambled) for 6-8 $\mathrm{h}$ and the medium was then changed to adipogenic induction medium, which was changed every 3 days. Untransfected cells were used as controls. RNA and protein were isolated on days $1,3,5$ and 7 . The transfection efficiency was analyzed by quantifying GFP expression using an inverted fluorescence microscope (LX70; Olympus, Tokyo, Japan) at $24 \mathrm{~h}$ after transduction. The interference efficiency was analyzed using the comparative $2^{-\Delta \Delta C \mathrm{CT}}$ method with $\beta$-actin as an endogenous control on day 3 in each group. Detailed information on the shRNA sequences is provided in Table I.

Analysis of cultured cells by flow cytometry. An analysis of cell surface molecules was performed on passage 3 cultures of rat BMSCs by flow cytometry with the following procedure: the medium was removed from the flasks and the cell layers were then washed twice with PBS and detached with $0.05 \%$ trypsin-EDTA (HyClone, Logan, UT, USA) for $2 \mathrm{~min}$ at room temperature. The BMSCs were collected by centrifugation (179 x g, at room temperature) and washed in flow cytometry buffer (BD Biosciences, Franklin Lakes, NJ, USA) consisting of $2 \%$ bovine serum albumin and $0.1 \%$ sodium azide in PBS. Subsequently, the cells were incubated with fluorescein-5-isothiocyanate (FITC)-conjugated monoclonal antibodies against CD44 (553133), CD45 (561867) and CD90 (561969), and phycoerythrin (PE)-conjugated antibody against CD34 (550619) (all from BD Biosciences). Following incubation with the antibodies for $30 \mathrm{~min}$ at $4^{\circ} \mathrm{C}$, the cells were washed with flow cytometry buffer. The washed cells were pelleted and resuspended in flow cytometry buffer containing $1 \%$ paraformaldehyde for $20 \mathrm{~min}$. Non-specific fluorescence was determined using equal aliquots of the cell preparation that were incubated with anti-mouse monoclonal antibodies. Data were acquired and analyzed on a FACSCalibur flow cytometer with CellQuest software (BD Biosciences).

$R N A$ isolation and reverse transcription-quantitative PCR $(R T-q P C R)$. Total RNA was extracted from the cells using TRIzol (Takara Bio, Inc., Tokyo, Japan) according to the manufacturer's instructions. RNA concentrations were assessed with the optical density at $260 \mathrm{~nm}$ (OD260) using a kinetic biospectrometer (Eppendorf AG, Hamburg, Germany). Reverse transcription reactions were completed using 300-500 ng of total RNA as input for the High Capacity cDNA Reverse Transcription kit (Applied Biosystems, Foster City, CA, USA). cDNA was generated from the total RNA using PrimeScript RT Master Mix (5X) (Takara Bio, Inc.) according to the manufacturer's instructions. To quantify mRNA expression, SYBR ${ }^{\circledR}$ Premix Ex Taq (2X) (Takara Bio, Inc.) was used. Briefly, each of the resulting eight reverse transcription pools containing cDNA template was diluted, mixed with Taq and loaded into each of the 8 fill ports on the TaqMan array. The 
Table I. Nucleotide sequences of the shRNAs for RNA interference.

\begin{tabular}{llll}
\hline Name & & shRNA primer sequence $\left(5^{\prime} \rightarrow 3^{\prime}\right)$ & Size $(\mathrm{bp})$ \\
\hline shEvi1-2 & F: GATCCGCAGTGAGGTCTGCCATAATTCAAGAGATTATGGCAGACCTCACTGCTTTTTTG & 59 \\
& R: AATTCAAAAAAGCAGTGAGGTCTGCCATAATCTCTTGAATTATGGCAGACCTCACTGCG & 59 \\
shEvi1-1 & F: GATCCGGAAGCAACATGGAAACAATTCAAGAGATTGTTTCCATGTTGCTTCCTTTTTG & 59 \\
& R: AATTCAAAAAAGGAAGCAACATGGAAACAATCTCTTGAATTGTTTCCATGTTGCTTCCG & 59 \\
\hline
\end{tabular}

F, forward; R, reverse.

Table II. Nucleotide sequences of the primers used for RT-qPCR.

\begin{tabular}{|c|c|c|c|}
\hline Gene & Primer sequence $\left(5^{\prime} \rightarrow 3^{\prime}\right)$ & Melting temperature & Product size (bp) \\
\hline \multirow[t]{2}{*}{$\beta$-actin } & F: GTGGAGTGCCCAAGCACCA & 52 & \multirow[t]{2}{*}{213} \\
\hline & R: CTCTAATGTCACGACGATTTC & 52 & \\
\hline \multirow[t]{2}{*}{ Evi1 } & F: AGGAGAAGAACCCTGTGGCTA & 57.8 & \multirow[t]{2}{*}{280} \\
\hline & R: GAGTCCTAAAAGCGCTGTCC & 57.45 & \\
\hline \multirow[t]{2}{*}{$\mathrm{OCN}$} & F: AATAGACTCCGGCGCTACCT & 57.45 & \multirow[t]{2}{*}{229} \\
\hline & R: AGCTGTGCCGTCCATACTTT & 55.4 & \\
\hline \multirow[t]{2}{*}{$\mathrm{OPN}$} & F: AGCCATGAGTCAAGTCAGCT & 55.85 & \multirow[t]{2}{*}{260} \\
\hline & R: ACTCGCCTGACTGTCGATAG & 57.8 & \\
\hline \multirow[t]{2}{*}{ BSP } & F: GCACGGTTGAGTATGGGGAA & 57.45 & \multirow[t]{2}{*}{260} \\
\hline & R: TGCACCTTCCTGAGTTGAGC & 57.45 & \\
\hline \multirow[t]{2}{*}{ LPL } & F: AGCTGACCAGTTATGGCACC & 57.8 & \multirow[t]{2}{*}{238} \\
\hline & R: ATCCTGACCCTCGTAGCCTT & 57.45 & \\
\hline \multirow[t]{2}{*}{$\operatorname{PPAR} \gamma 2$} & F: GCTGCAGCGCTAAATTCATCT & 58.66 & \multirow[t]{2}{*}{195} \\
\hline & R: GGGAGTGGTCATCCATCACAG & 59.76 & \\
\hline
\end{tabular}

Evi1, ectopic viral integration site-1; OCN, osteocalcin; OPN, osteopontin; BSP, bone sialoprotein; LPL, lipoprotein lipase; PPAR $\gamma 2$, peroxisome proliferator-activated receptor $\gamma 2$; F, forward; R, reverse.

card was centrifuged once for $1 \mathrm{~min}$ at $331 \mathrm{x}$ g to distribute samples to the multiple wells on the array, then sealed to prevent well-to-well contamination. Finally, the cards were processed and analyzed in the CFX96 ${ }^{\mathrm{TM}}$ Real-Time PCR Detection System (Bio-Rad, Hercules, CA, USA) with an initial denaturation step at $95^{\circ} \mathrm{C}$ for $30 \mathrm{sec}$ followed by 40 cycles of denaturation for $5 \mathrm{sec}$ at $95^{\circ} \mathrm{C}$, annealing and extension for $30 \mathrm{sec}$ at $60^{\circ} \mathrm{C}$. We compared the relative mRNA levels by the comparative $2^{-\Delta \Delta C T}$ method with $\beta$-actin as an endogenous control for RT-qPCR in each group on days 1, 3, 5 and 7. Detailed information on PCR, including the primer sequences, is provided in Table II.

Protein isolation and western blot analysis. The cells were plated into 60-mm dishes in 10\% FBS-DMEM and cultured until nearly $90 \%$ confluence. Subsequently, they were washed twice with ice-cold PBS and then harvested with cell lysis buffer containing Protease Inhibitor Cocktail (Complete ${ }^{\mathrm{TM}}$; Roche Diagnostics GmbH, Mannheim, Germany) and $1 \mathrm{mM}$ phenylmethylsulfonyl fluoride (PMSF). Upon centrifugation at $13,000 \mathrm{x} \mathrm{g}$ for $5 \mathrm{~min}$ at $4^{\circ} \mathrm{C}$, the supernatants were collected and the total protein concentrations were determined using the BCA Protein Assay kit (Beyotime, Shanghai, China) following the manufacturer's instructions. SDS-PAGE $(10 \%)$ gels were prepared and 5-10 mg/lane of cellular proteins were loaded. The resolved proteins were transferred onto a polyvinylidene difluoride (PVDF) membrane and incubated (1:200 dilution) with primary antibodies [bone sialoprotein (BSP; SC-292394), osteocalcin (OCN; SC-30044) and lipoprotein lipase (LPL; SC-32885): rabbit polyclonal antibody; osteopontin (OPN; SC-21742) and peroxisome proliferator-activated receptor $\gamma 2$ (PPAR $\gamma 2$; SC-7273): mouse monoclonal antibody; Santa Cruz Biotechnology, Inc., Santa Cruz, CA, USA] and incubated (1:1,000 dilution) with $\beta$-actin (BM0627; mouse monoclonal antibody; Wuhan Boster Biological Technology, Ltd., Hubei, China) following the manufacturer's instructions. Following incubation (1:5,000 dilution) with HRP-conjugated secondary antibodies (goat anti-rabbit or goat anti-mouse IgG; Wuhan Boster Biological Technology, Ltd.), the blotting bands were visualized using the ECL Chemiluminescence kit (Thermo Fisher Scientific, Inc., Waltham, MA, USA) and quantified using the ChemiDoc ${ }^{\mathrm{TM}}$ XRS+ Imaging System (Bio-Rad). 

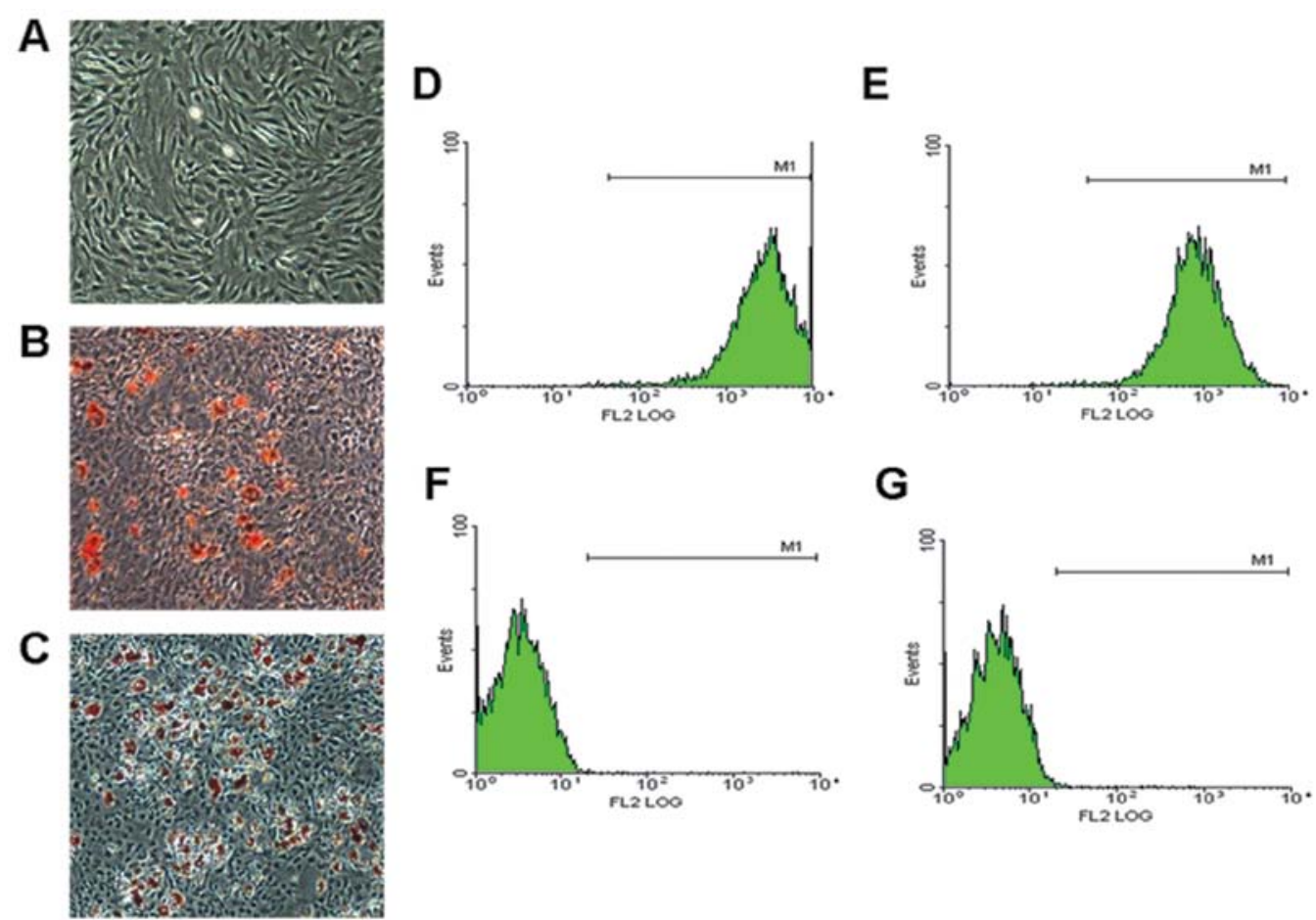

Figure 1. Microphotographs and flow cytometry of cultured bone mesenchymal stem cells (BMSCs). (A) Microphotograph of normal BMSCs on day 7 of differentiation. Differentiation of cultured BMSCs was monitored with (B) Oil Red O staining of lipid droplets on the 16th day of adipocyte differentiation and $(C)$ alizarin red staining of calcium deposition in the extracellular matrix on the 16th day of osteoblast differentiation. Original magnification, $\mathrm{x} 40$. Flow cytometry was used to array cultured BMSCs with (D and E) fluorescein-5-isothiocyanate (FITC)-CD90 and -CD44 and (F and G) phycoerythrin (PE)-CD34 and FITC-CD45.
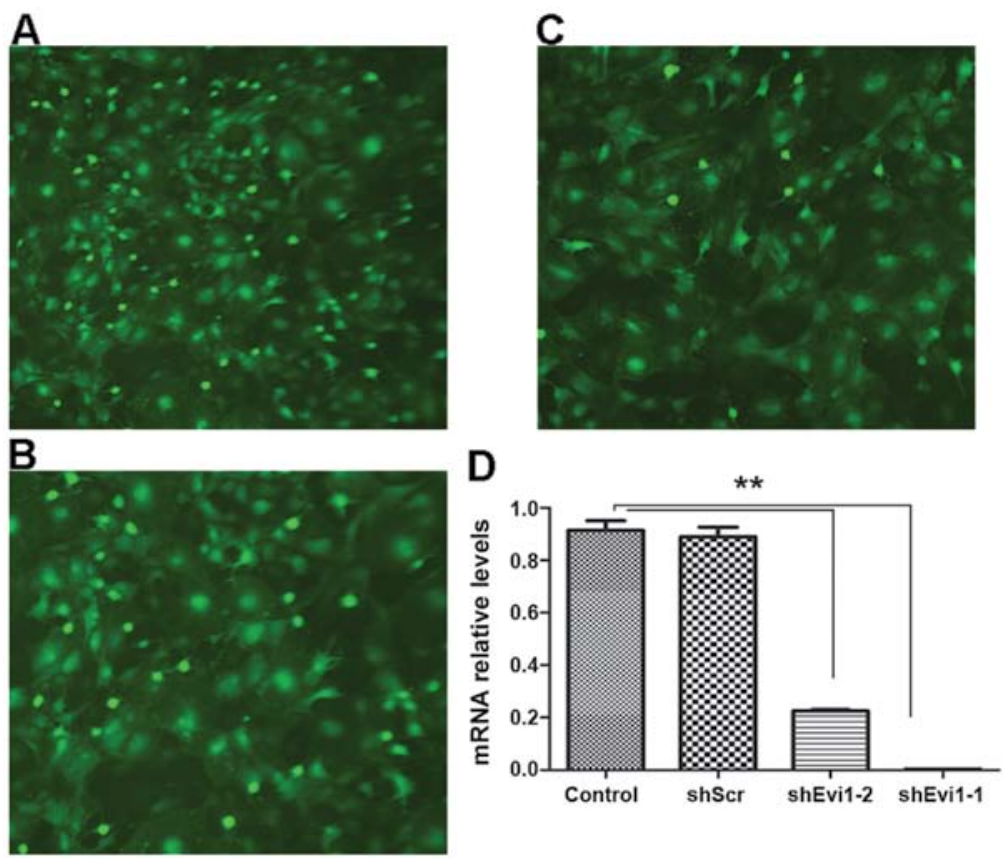

Figure 2. Microphotographs of transfections and effect of ectopic viral integration site-1 (Evil) short hairpin RNA (shRNA). Microphotographs of bone mesenchymal stem cells (BMSCs) following treatment with Ad-shEvil or siScr (scrambled) by fluorescence microscopy $24 \mathrm{~h}$ after transduction. (A) Ad-shEvil-1 group, (B) Ad-shEvil-2 group and (C) scrambled group (Ad-shScr). Original magnification, x40. (D) The mRNA level of Evil in adipocyte derived from BMSCs at day 3 was measured by RT-qPCR. The data are representative of 3 independent experiments. The two sh-Evi1, Ad-shEvi1-1 and Ad-shEvi1-2, were used in the experiment. The Ad-shScr was used in the mock group. Control, untransfected cells. ${ }^{* *} \mathrm{P}<0.01$.

Statistical analysis. Statistical analysis was performed using SPSS 13.0 software. Significant differences between the study groups were determined using statistical methods [e.g., one-way analysis of variance (ANOVA) or the Student's t-test where appropriate]. A P-value $<0.05$ was considered to indicate a statistically significant difference. 


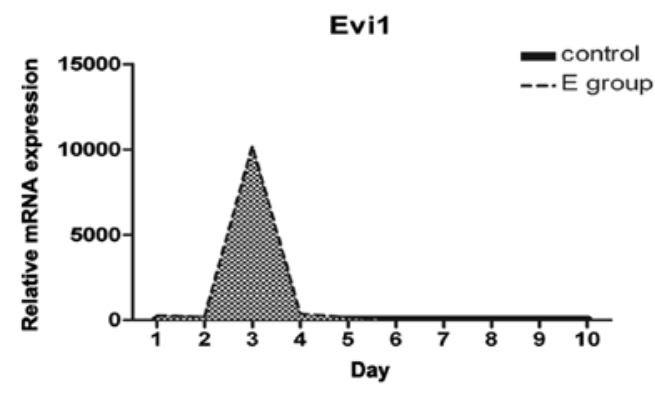

Figure 3. Ectopic viral integration site-1 (Evi1) is expressed early during the adipocyte differentiation of bone mesenchymal stem cells (BMSCs). Evi1 mRNA levels were measured by RT-qPCR during the first 10 days of the adipocyte differentiation of BMSCs (E group); normal cultured BMSCs were used as the control. The data are representative of 3 independent experiments.

\section{Results}

BMSC morphology and characteristics. The BMSCs were obtained from the BM of 4-week-old SD rats maintained a fibroblast-like morphology under monolayer culture conditions (Fig. 1A), and were capable of differentiating into adipocytes (Fig. 1B) and osteogenic cells (Fig. 1C) under specific induction. Flow cytometric analysis revealed that the BMSC populations were positive for the MSC markers, CD90 and CD44 (Fig. 1D and E), but were negative for the hematopoietic markers, CD34 and CD45 (Fig. 1F and G). These results demonstrated that we obtained the correct rat BMSCs.

Increased Evil expression at the onset of BMSC adipogenesis. We examined the differences and changes occurring in gene expression profiles between rat BMSCs during the process of adipogenesis and normal BMSCs over the first 10 days.
Evi1 expression was dynamically regulated during adipocyte differentiation. Evil mRNA levels were low in the normal BMSCs (control), with a peak in expression being observed at confluence and early after hormone induction, and with moderate expression levels during the later stages of differentiation (Fig. 3). Evil expression was significantly higher in the rat BMSCs undergoing adipogenic differentiation than in the normal BMSCs. This finding suggests that the increased Evi1 expression in differentiating BMSCs during the early stages plays a critical role in the adipogenic process.

Evil knockdown using Ad-shEvil expression vector. We created an adenoviral vector encoding shRNA against Evil (Evil shRNA) for expressing Evil siRNA to knockdown the Evil gene at the onset of BMSC adipogenesis. We used two different siRNA sequences to target the rat Evil gene, termed Ad-shEvi1-1 (KD1) (Fig. 2A) and Ad-shEvi1-2 (KD2) (Fig. 2B). The cells in the mock group were transfected with an adenoviral vector expressing scrambled shRNA Ad-shScr (Fig. 2C). KD1 and KD2 markedly reduced Evil expression at the mRNA level (Fig. 2). The inhibition rate of KD1 and KD2 was 90.9 and $72.3 \%$, respectively, in the BMSCs undergoing adipogenic differentiation compared to the mock (scrambled) and control (untransfected) groups at the mRNA level (Fig. 2D).

Effects of Evil shRNA on adipogenic and osteogenic differentiation of BMSCs. As mentioned above, in two representative subclones, infection with Evil shRNA-1 caused a more profound reduction in the expression of the Evil gene than infection with Evil shRNA-2. Hereafter, we mainly used the Ad-shEvil-1 subline for further analysis. The expression levels of specific osteogenic and adipogenic genes were measured by RT-qPCR (Fig. 4) and western blot analysis (Figs. 5 and 6) on days $1,3,5$ and 7 post-transduction. Our results revealed that
BSP
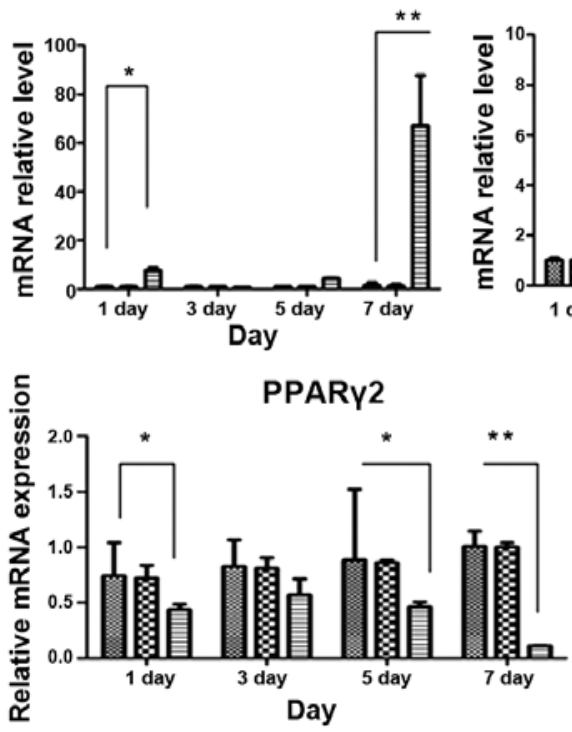

OPN
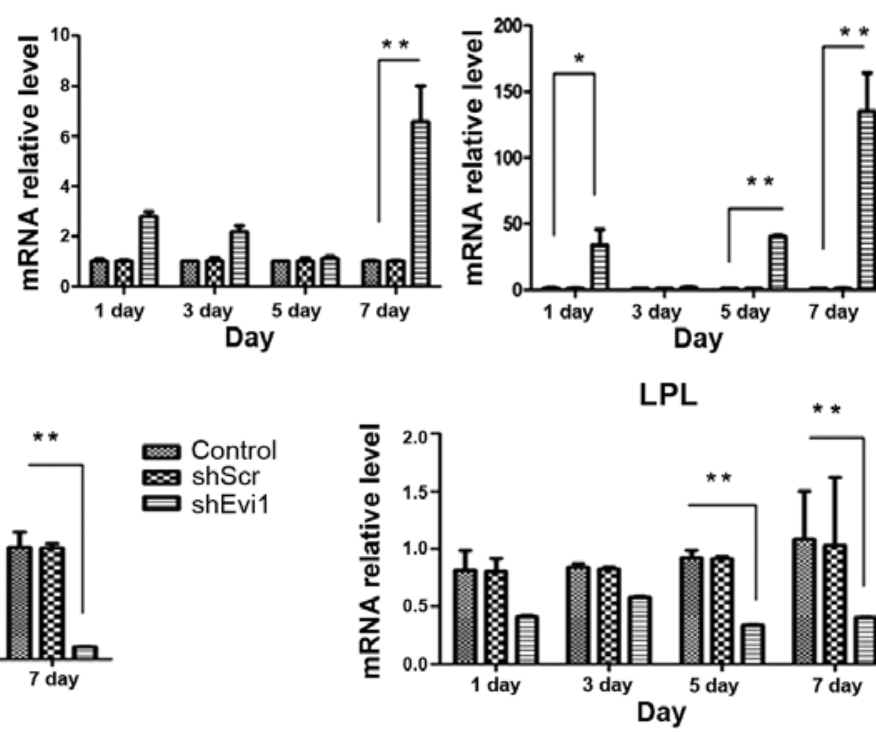

OCN
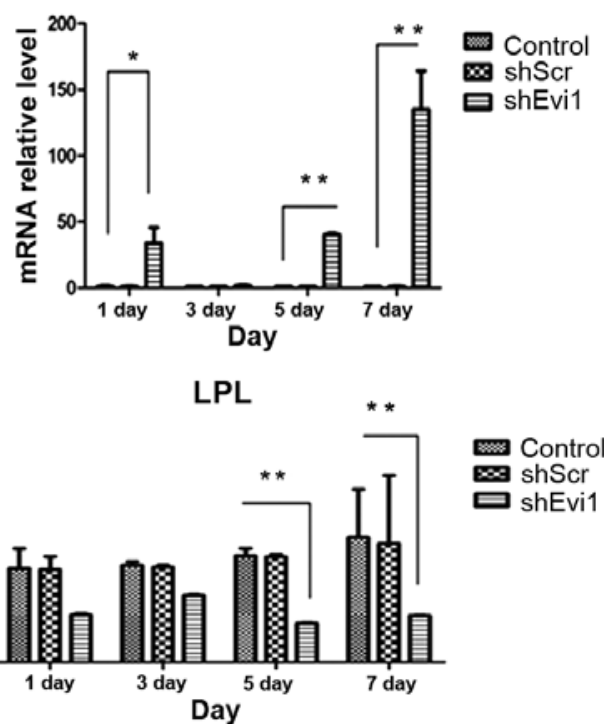

Figure 4. The mRNA levels of specific osteogenic and adipogenic genes in each group. mRNA expression was evaluated by RT-qPCR on days 1, 3, 5, and 7 post-transduction. Adipocyte differentiation of the bone mesenchymal stem cell (BMSC) group was used as a control. The specific osteogenic markers, markers sialoprotein (BSP), osteopontin (OPN) and osteocalcin (OCN), were upregulated in the Ad-shEvil-transduced BMSCs, particularly on day 7. The specific adipogenic markers, peroxisome proliferator-activated receptor $\gamma 2$ (PPAR $\gamma 2$ ) and lipoprotein lipase (LPL), were downregulated in the Ad-shEvi1-transduced BMSCs (mean \pm SEM, $\mathrm{n}=3$ experiments). ${ }^{*} \mathrm{P}<0.05 ;{ }^{* *} \mathrm{p}<0.01$. 

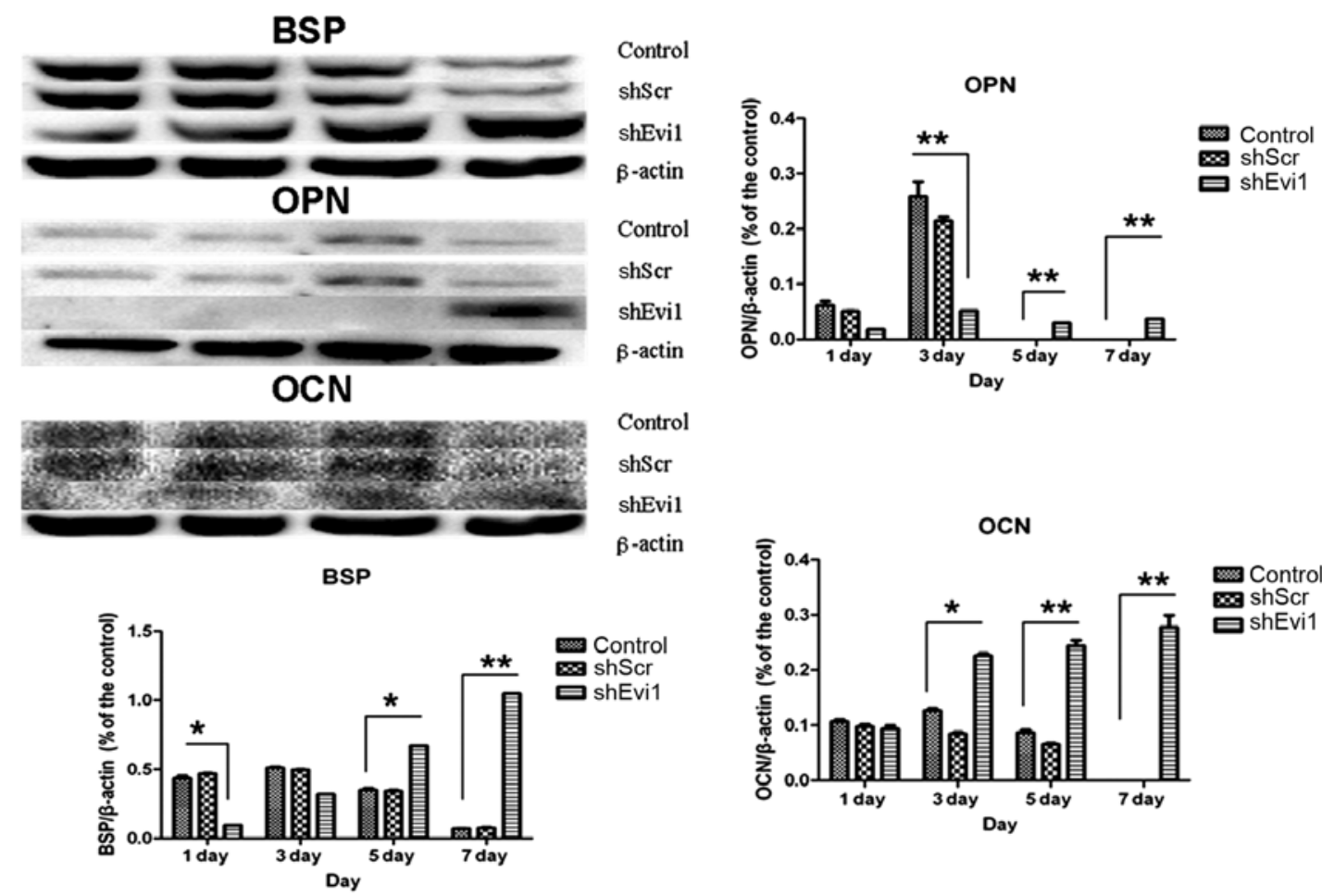

Figure 5. The protein levels of specific osteogenic genes. Protein expression was evaluated by western blot analysis on days 1, 3, 5, and 7 post-transduction Osteogenic differentiation of the bone mesenchymal stem cell (BMSC) group was used as a control. The data are representative of 3 independent experiments. The specific osteogenic markers, sialoprotein (BSP), osteopontin (OPN) and osteocalcin (OCN), were upregulated in the Ad-shEvil-transduced BMSCs compared with the control group (mean $\pm \mathrm{SEM}, \mathrm{n}=3$ experiments). ${ }^{*} \mathrm{P}<0.05 ;{ }^{* *} \mathrm{p}<0.01$.
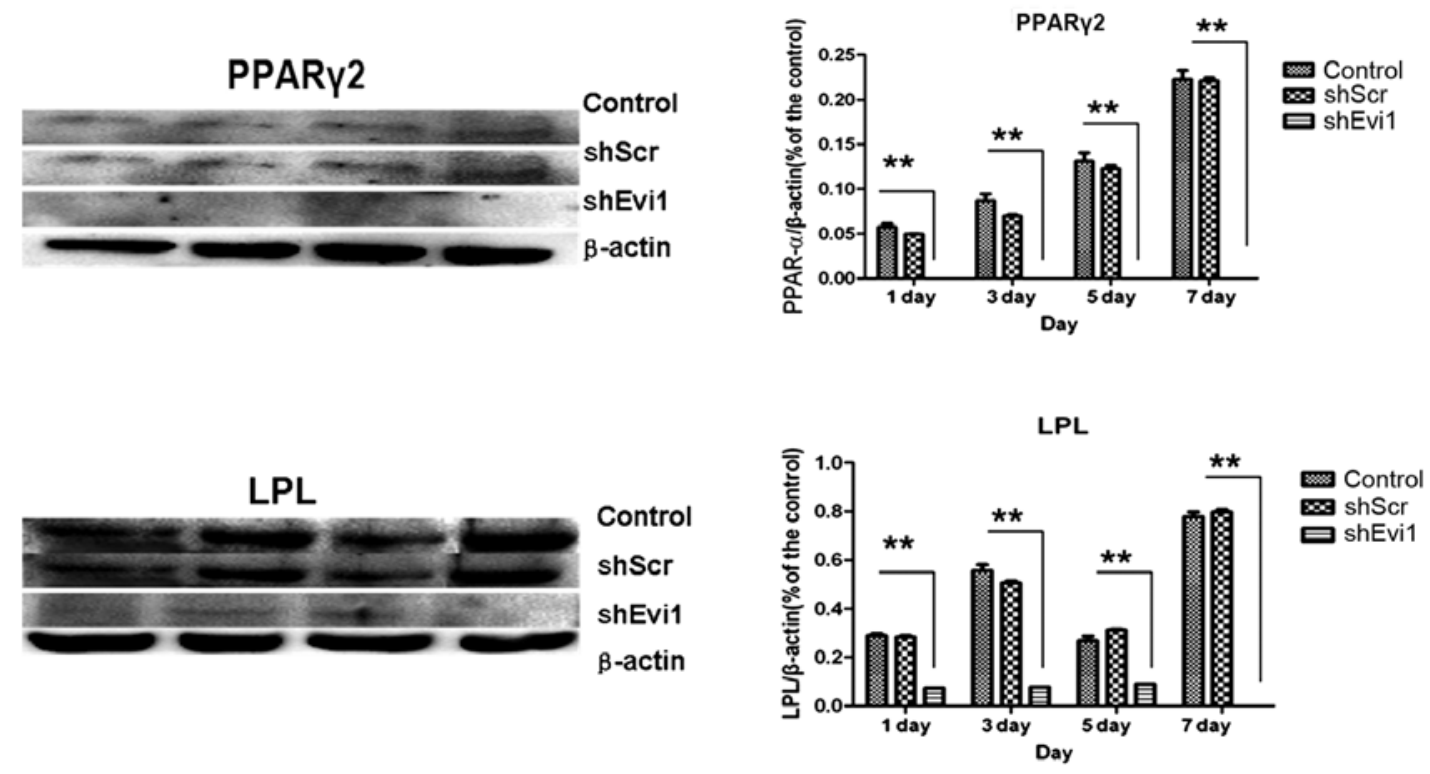

Figure 6. The protein levels of specific adipogenic genes. Protein expression was evaluated by western blot analysis on days 1, 3, 5, and 7 post-transduction. Adipocyte differentiation of the bone mesenchymal stem cell (BMSC) group was used as a control. The data are representative of 3 independent experiments. The specific adipogenic markers, peroxisome proliferator-activated receptor $\gamma 2$ (PPAR $\gamma 2$ ) and lipoprotein lipase (LPL), were downregulated in the Ad-shEvi1-transduced BMSCs (mean \pm SEM, $\mathrm{n}=3$ experiments). ${ }^{* *} \mathrm{P}<0.01$.

the specific osteogenic markers, BSP, OPN and OCN, were downregulated during the adipogenesis of rat BMSCs and were upregulated in the Ad-shEvi1-transduced BMSCs at both the mRNA and protein level, particularly on day 7 (Figs. 4 and 5). The specific adipogenic markers, PPAR $\gamma 2$ and LPL, were downregulated in the Ad-shEvi1-transduced BMSCs in comparison to the BMSCs undergoing adipogenic differentiation at both the mRNA and the protein level (Figs. 4 and 6). Statistical analysis indicating that knocking down the expression of Evil by Ad-shEvil significantly inhibited the adipogenic 
differentiation of BMSCs and simultaneously enhanced the osteogenic differentiation of BMSCs.

\section{Discussion}

In this study, we substantiated that Evil was highly expressed during the of adipogenesis of rat BMSCs, and that the Evi1 expression level markedly increased on day 3 of adipogenic differentiation following the addition of adipogenic inducers. The experimental data further verified that the silencing of Evil by RNAi in the BMSCs inhibited adipogenic differentiation, while it promoted osteogenic differentiation. The inhibition of Evil may thus represent a therapeutic strategy for promoting bone formation.

Primary OP is a polygenetic disease characterized by low bone mineral density and microarchitectural deteriorations, leading to an increased risk of fragility fractures of the vertebrae, the femoral neck and other typical localizations of lower incidence (19). As OP is characterized by pathologically enhanced osteoclast development and function, researchers over the past decaces have mainly focused on the imbalance of bone resorption and bone formation (20). Therefore, anti-resorptive treatment targeting mature osteoclasts and osteoclastogenesis to promote the RANK/RANKL pathway, has evolved as a standard therapy over the past decades $(4,19,21)$. However, a considerable number of clinical trials on OP have demonstrated that anti-resorptive treatments lead to a reduction in bone remodeling and turnover and are assoicated with a series of adverse events (22-24). Research on presumptive deficiencies in bone anabolism has been neglected. The importance of the balance between bone formation and bone resorption in OP prompted us to focus on bone formation rather than bone resorption.

A theoretical inverse association ex ists between osteogenic and adipogenic lineage commitment and differentiation, indicating that differentiation towards an osteoblast phenotype occurs at the expense of an adipocytic phenotype $(25,26)$. Previous studies have revealed enhanced BMSC differentiation into adipocytes in the BM of post-menopausal women. All the aforementioned research results led us to hypothesize that the inhibition of adipogenesis in stem cells may alleviate OP using the simultaneous stimulation of osteogenesis, which was confirmed by the results of the present study. In addition, our data validated the results of Gimble and Nuttall, as well as those of Beresford et al, who demonstrated in vitro that the balance between BMSC adipogenic and osteogenic processes exhibits an inverse or reciprocal association $(27,28)$.

The Evil isoform of MECOM is a member of the PR (PRDI-BF1 and RIZ homology) domain-containing family of zinc finger transcriptional regulatory proteins and is closely related in sequence and structure to Prdm16 (15). Previous studies have revealed that Evi1 is not expressed or is expressed at low levels in normal BM (28). In this study, we found that Evil expression increased at the onset of adipogenesis of rat BMSCs, and that the mRNA level of Evil was low in normal growing BMSCs. Taken together, these results indicate that the increased expression of Evil in early differentiating BMSCs is critical to the adipogenic process.

Our data validated Evil gene expression in the process of BMSC adipogenic differentiation using mature adipogenic induction medium at the cellular level. Our results revealed that the Evil gene was not only expressed in the first 5 days after the addition of adipogenic inducers, but that its mRNA expression reached peak levels on day 3. We also measured the protein expression level of Evi1, but we were unable to produce a result. In order to determine whether the high expression of Evil determines the adiopogenic lineage commitment, we further measyred the RNA and protein levels of adipogenic and osteogenic markers.

We observed a lower expression of the adipogenic markers, PPAR $\gamma 2$ and LPL, in the RNAi group in comparison to the adipocyte differentiation group on days 1,3,5, and 7 of differentiation. Furthermore, our results revealed a higher expression of the osteogenic markers, BSP, OPN and OCN, in the RNAi group in comparison to the adipocyte differentiation group on days 1, 3, 5, and 7 of differentiation. Notably, the levels of the markers, BSP, OPN, OCN, PPAR $\gamma 2$ and LPL, varied markedly on day 7. This phenomenon can be explained by the fact that the expression of the Evil gene increased in the first 5 days of BMSC adipogenesis (Fig. 3) and that the corresponding osteogenic and adipogenic markers as the Evil downstream genes possess hysteresis. The mechanisms involved remain to be elucidated. It will now be important to examine the role of Evil in estrogen deficiency-induced OP using gain- and-loss-of-function studies on animals.

Evil is highly expressed in certain cytogenetic subsets of adult acute myeloid leukaemia (AML), and has been associated with inferior survival (29). Consequently, we presume that the side-effects caused by knocking down the Evil gene are relatively limited.

In conclusion, in this study, we identified Evi1 as a key competency factor that allows rat BMSCs to undergo adipogenesis. While animals are not exactly the same as humans, it is logical to make the inference that an effect observed in rats may also apply to humans. These findings indicate that the suppression of the Evil gene prevents estrogen deficiency-induced bone loss by the simultaneous stimulation of osteogenesis and the inhibition of adipogenesis.

\section{References}

1. Weinstein RS, Jilka RL, Parfitt AM and Manolagas SC: Inhibition of osteoblastogenesis and promotion of apoptosis of osteoblasts and osteocytes by glucocorticoids. Potential mechanisms of their deleterious effects on bone. J Clin Invest 102: 274-282, 1998.

2. Morrison NA, Qi JC, Tokita A, Kelly PJ, Crofts L, Nguyen TV, Sambrook PN and Eisman JA: Prediction of bone density from vitamin D receptor alleles. Nature 367: 284-287, 1994.

3. Seeman E: Bone quality: The material and structural basis of bone strength. J Bone Miner Metab 26: 1-8, 2008.

4. Pietschmann P, Rauner M, Sipos W and Kerschan-Schindl K: Osteoporosis: An age-related and gender-specific disease - a mini-review. Gerontology 55: 3-12, 2009.

5. Kassem M and Abdallah BM: Human bone-marrow-derived mesenchymal stem cells: Biological characteristics and potential role in therapy of degenerative diseases. Cell Tissue Res 331: 157-163, 2008.

6. Egermann M, Heil P, Tami A, Ito K, Janicki P, Von Rechenberg B, Hofstetter W and Richards PJ: Influence of defective bone marrow osteogenesis on fracture repair in an experimental model of senile osteoporosis. J Orthop Res 28: 798-804, 2010.

7. Rodriguez JP, Astudillo P, Rios S and Pino AM: Involvement of adipogenic potential of human bone marrow mesenchymal stem cells (MSCs) in osteoporosis. Curr Stem Cell Res Ther 3: 208-218, 2008.

8. Pittenger MF, Mackay AM, Beck SC, Jaiswal RK, Douglas R, Mosca JD, Moorman MA, Simonetti DW, Craig S and Marshak DR: Multilineage potential of adult human mesenchymal stem cells. Science 284: 143-147, 1999. 
9. Uejima S, Okada K, Kagami H, Taguchi A and Ueda M: Bone marrow stromal cell therapy improves femoral bone mineral density and mechanical strength in ovariectomized rats. Cytotherapy 10: 479-489, 2008.

10. Ocarino Nde M, Boeloni JN, Jorgetti V, Gomes DA, Goes AM and Serakides R: Intra-bone marrow injection of mesenchymal stem cells improves the femur bone mass of osteoporotic female rats. Connect Tissue Res 51: 426-433, 2010.

11. Lien CY, Chih-Yuan Ho K, Lee OK, Blunn GW and Su Y: Restoration of bone mass and strength in glucocorticoid-treated mice by systemic transplantation of CXCR 4 and cbfa- 1 co-expressing mesenchymal stem cells. J Bone Miner Res 24 837-848, 2009.

12. Park S-H, Sim WY, Min B-H, Yang SS, Khademhosseini A and Kaplan DL: Chip-based comparison of the osteogenesis of human bone marrow- and adipose tissue-derived mesenchymal stem cells under mechanical stimulation. PLoS One 7: e46689, 2012.

13. Mimeault M and Batra SK: Recent progress on tissue-resident adult stem cell biology and their therapeutic implications. Stem Cell Rev 4: 27-49, 2008.

14. Rodríguez JP, Garat S, Gajardo H, Pino AM and Seitz G: Abnormal osteogenesis in osteoporotic patients is reflected by altered mesenchymal stem cells dynamics. J Cell Biochem 75 414-423, 1999.

15. Ishibashi J, Firtina Z, Rajakumari S, Wood KH, Conroe HM, Steger DJ and Seale P: An Evi1-C/EBP $\beta$ complex controls peroxisome proliferator-activated receptor $\gamma 2$ gene expression to initiate white fat cell differentiation. Mol Cell Biol 32: 2289-2299, 2012.

16. Yue B, Lu B, Dai KR, Zhang XL, Yu CF, Lou JR and Tang TT: BMP2 gene therapy on the repair of bone defects of aged rats. Calcif Tissue Int 77: 395-403, 2005.

17. Fu L, Tang T, Miao Y, Zhang S, Qu Z and Dai K: Stimulation of osteogenic differentiation and inhibition of adipogenic differentiation in bone marrow stromal cells by alendronate via ERK and JNK activation. Bone 43: 40-47, 2008.

18. Liang J, Liu C, Qiao A, Cui Y, Zhang H, Cui A, Zhang S, Yang Y, Xiao X, Chen Y, et al: MicroRNA-29a-c decrease fasting blood glucose levels by negatively regulating hepatic gluconeogenesis. J Hepatol 58: 535-542, 2013.
19. Rachner TD, Khosla S and Hofbauer LC: Osteoporosis: Now and the future. Lancet 377: 1276-1287, 2011.

20. Manolagas SC: Birth and death of bone cells: Basic regulatory mechanisms and implications for the pathogenesis and treatment of osteoporosis. Endocr Rev 21: 115-137, 2000.

21. Khosla S: Minireview: The OPG/RANKL/RANK system. Endocrinology 142: 5050-5055, 2001.

22. Schwartzman J and Yazici Y: Denosumab in postmenopausal women with low bone mineral density. N Engl J Med 354: 2390-2391; author reply 2390-2391, 2006.

23. Watts NB, Roux C, Modlin JF, Brown JP, Daniels A, Jackson S, Smith S, Zack DJ, Zhou L, Grauer A, et al: Infections in postmenopausal women with osteoporosis treated with denosumab or placebo: Coincidence or causal association? Osteoporos Int 23: 327-337, 2012.

24. Sobacchi C, Frattini A, Guerrini MM, Abinun M, Pangrazio A, Susani L, Bredius R, Mancini G, Cant A, Bishop N, et al: Osteoclast-poor human osteopetrosis due to mutations in the gene encoding RANKL. Nat Genet 39: 960-962, 2007.

25. James AW, Pang S, Askarinam A, Corselli M, Zara JN, Goyal R, Chang L, Pan A, Shen J, Yuan W, et al: Additive effects of sonic hedgehog and Nell-1 signaling in osteogenic versus adipogenic differentiation of human adipose-derived stromal cells. Stem Cells Dev 21: 2170-2178, 2012.

26. Pei L and Tontonoz P: Fat's loss is bone's gain. J Clin Invest 113 805-806, 2004.

27. Gimble JM and Nuttall ME: The relationship between adipose tissue and bone metabolism. Clin Biochem 45: 874-879, 2012.

28. Beresford JN, Bennett JH, Devlin C, Leboy PS and Owen ME: Evidence for an inverse relationship between the differentiation of adipocytic and osteogenic cells in rat marrow stromal cell cultures. J Cell Sci 102: 341-351, 1992.

29. Ho PA, Alonzo TA, Gerbing RB, Pollard JA, Hirsch B, Raimondi SC, Cooper T, Gamis AS and Meshinchi S: High EVI1 expression is associated with MLL rearrangements and predicts decreased survival in paediatric acute myeloid leukaemia: A report from the children's oncology group. Br J Haematol 162: 670-677, 2013. 